\title{
SPARSITY AND UNIQUENESS FOR SOME SPECIFIC UNDER-DETERMINED LINEAR SYSTEMS.
}

\author{
Jean-Jacques FUCHS
}

\author{
IRISA/Université de Rennes I \\ Campus de Beaulieu - 35042 Rennes Cedex - France \\ fuchs@irisa.fr
}

\begin{abstract}
The purpose of this contribution is to extend some results on sparse representations of signals in redundant bases developed for arbitrary bases to two frequently encountered bases.

The general problem is the following: given a $n \times m$ matrix $A$ with $m>n$ and a vector $b=A x_{o}$ with $x_{o}$ having $q<n$ nonzero components, find sufficient conditions for $x_{o}$ to be the unique sparsest solution to $A x=A x_{o}$. The answer gives an upper-bound on $q$ depending upon $A$.

We consider the cases where $A$ is a Vandermonde matrix or a real Fourier matrix and the components of $x_{o}$ are known to be greater than or equal to zero. The sufficien$\mathrm{t}$ conditions we get are much weaker than those valid for arbitrary matrices and guarantee further that $x_{o}$ can be recovered by solving a linear program.
\end{abstract}

\section{INTRODUCTION}

The problem of the recovery of exact sparse solutions of a set of under-determined linear equations has been proposed in [1] and has received some attention since $[2,3,4,5,6]$. The results obtained so far are as follows.

Given a (n,m)-matrix $A$ with $m>n$ and a vector $b$ that indeed admits an exact sparse representation, say $b=A x_{o}$, it has been shown that if the number of non-zero entries in $x_{o}$ is smaller than a given bound, then $x_{o}$ is the unique sparsest representation. Since searching for the sparses$\mathrm{t}$ representation is a NP hard problem [7] that can only be solved by exhaustive search, one is tempted to replace the true search for the sparsest solution

$$
\min _{x}\|x\|_{0} \quad \text { subject to } A x=b,
$$

with $\|x\|_{0}$ the number of non-zero entries in $x$, by the easyto-solve linear program

$$
\min _{x}\|x\|_{1} \quad \text { subject to } A x=b,
$$
with $\|x\|_{1}=\sum_{1}^{m}\left|x_{j}\right|$, i.e., to minimize the $\ell_{1}$ norm of $x$ instead of the sparsity itself. The problem is then to determine sufficient conditions for the two criteria to have the same unique solution.

In order to define when this is the case, one generally normalizes the columns $a_{j}$ of $A$ to one in euclidean norm and introduces the mutual coherence [1]

$$
M=\max _{1 \leq i \neq j \leq m}\left|\boldsymbol{q}^{T} a_{j}\right|,
$$

of the dictionary whose atoms or components are the columns of $A$. The smaller $M$, the less coherent are the components of the dictionary and $M=0$ if and only if the columns are orthogonal. It is worthwhile to know that there are indeed redundant dictionaries with $m \simeq n^{2}$ components and mutual coherence $M \simeq \frac{1}{\sqrt{n}}[8,9]$.

It has been shown in $[1,2,3,4]$ that if

$$
\left\|x_{o}\right\|_{0}<\frac{1}{2}\left(1+\frac{1}{M}\right)
$$

then $x_{o}$ is the unique sparsest representation of $b$ and that it can be recovered by solving (LP) defined above. It is worth noting that (2) is independent of the magnitudes of the nonzero entries of $x_{o}$. Being able to recover $x_{o}$ appears to be only a matter of structure, of angles between vectors. This is similar to what happens with identifiability conditions in estimation theory or observability conditions in systems theory.

Here we will obtain stronger results for two specific $A$ matrices and when the weights in $x_{o}$ are known to be greater than or equal to zero. This last assumption changes the nature of the problem and the conditions we will obtain will be independent of $m$ the number of columns in $A$.

We will consider the Vandermonde matrices whose column$\mathrm{s}$ are of the form

$$
v(\alpha)=\left[\begin{array}{lllllll}
1 & \alpha & \alpha^{2} & \ldots & \alpha^{k} & \ldots & \alpha^{n-1}
\end{array}\right]^{T}
$$

and real Fourier matrices whose columns are

$$
\begin{aligned}
& s(\omega) \quad=\frac{1}{2}\left(v\left(e^{i \omega}\right)+v\left(e^{-i \omega}\right)\right) \\
& =\left[\begin{array}{lllllll}
1 & \cos \omega & \cos 2 \omega & \ldots & \cos k \omega & \ldots & \cos (n-1) \omega
\end{array}\right]^{T} .
\end{aligned}
$$

As in [4] we will start from the optimality conditions satisfied by an optimum of the linear program $(L P)$ and the sufficient conditions insuring uniqueness of the optimum. We will see that it is no longer useful to consider $A$ matrices whose columns have unit euclidean norm, i.e. we will not normalize the vectors $v(\alpha)$ and $s(\omega)$ defined above. 


\section{OPTIMALITY CONDITIONS FOR (LP)}

Since we assume the weights in $x$ to be greater than or equal to zero, the linear program (LP) minimizing the $\ell_{1}$-norm becomes

$$
\min _{x} \mathbf{1}^{T} x \text { subject to } A x=b \text { and } x \geq 0
$$

where 1 denotes a column vector of ones of adequate dimension. The dual of this linear program is [10] :

$$
\max _{d} b^{T} d \text { subject to } A^{T} d \leq \mathbf{1}
$$

(DLP+)

For given $b$, let $x_{o} \geq 0$ be a feasible point of (LP+), we denote $\bar{x}_{o}$ the reduced dimensional vector built with the strictly positive components in $x_{o}$ and $\bar{A}_{o}$ the matrix built with the corresponding columns of $A$, one thus has $A x_{o}=$ $\bar{A}_{o} \bar{x}_{o}=b$. Standard duality theory for convex programs then says that the point $x_{o}$ is an optimum of (LP+) if and only if there exists, say $d_{o}$, a dual feasible point that achieves the same cost, i.e.

$$
\exists d_{o} \ni A^{T} d_{o} \leq \mathbf{1} \text { and } \mathbf{1}^{T} x_{o}=b^{T} d_{o}
$$

This point is then an optimum of (DLP+). Furthermore both optima are unique if no constraint of the dual is degenerate, i.e., $a_{j}^{T} d_{o}<1 \quad \forall a_{j} \notin \bar{A}_{o}$. To summarize one has the following proposition.

Lemma 1: The point $x_{o}$ is the unique optimum of (LP+) if

$$
\begin{array}{r}
\exists d_{o} \ni \quad \mathbf{1}^{T} x_{o}=b^{T} d_{o}, \bar{A}_{o}^{T} d_{o}=\mathbf{1} \\
\quad \text { and } \quad a_{j}^{T} d_{o}<1, \forall a_{j} \notin \bar{A}_{o}
\end{array}
$$

This is only a sufficient condition for uniqueness but since the solution of the primal linear program (LP+) is degenerate, i.e. $\left\|x_{o}\right\|_{0}<n$, one cannot expect a stronger result. When the optimum of the primal is degenerate the optimum of the dual (DLP+) is undetermined and this also signifies that the primal is unstable, i.e., even the slightest perturbation of $b$ will lead to a drastic change in the optimum of the primal. The optimum of the primal will loose its sparsity in a fully unpredictable way.

In the sequel we will apply Lemma 1 to the two specific $A$ matrices whose columns have been introduced above (3), (4). In [4], it is applied to arbitrary matrices and leads to condition (2).

\section{THE VANDERMONDE MATRIX CASE}

\subsection{Sufficient condition for recovery}

The (n,m)-matrix $A$ has $m$ columns denoted $v(\alpha)$ defined in (3) with $\alpha$ having $m$ distinct values $\alpha_{i}$ taken for instance on a regular grid in $[-1,1[$. We seek sufficient conditions on $A$ and $\left\|x_{o}\right\|_{o}$, under which the solution of (LP+) with $b=A x_{o}$ is unique and equal to $x_{o}$ which is assumed to satisfy $x_{o} \geq 0$. It happens that the conditions we will get is independent of $m$ and on the way the $m$ distinct values $\alpha_{i}$ are taken. It only depends upon $n$ the number of observations. We prove the following results.

Lemma 2: For $A$ an (n,m)-Vandermonde-matrix with distinct columns, the sparsest solution to $\{A x=b, x \geq 0\}$ and to (LP+) is unique if $b=A x_{o}$ with $x_{o} \geq 0$ and

$$
\left\|x_{o}\right\|_{0} \leq\left\lfloor\frac{n-1}{2}\right\rfloor
$$

where $\lfloor z\rfloor$ denotes the nearest integer that is $\leq z$.

Proof: We indicate how to construct a vector $d_{o}$ satisfying (5). With an arbitrary vector $f=\left[\begin{array}{llll}f_{0} & f_{1} & \ldots & f_{n-1}\end{array}\right]^{T}$ we associate the polynomial $F(\alpha)=f^{T} v(\alpha)=f_{0}+f_{1} \alpha+. .+$ $f_{n-1} \alpha^{n-1}$. The idea is to build a polynomial in $\alpha$ that is zero on the values $\alpha_{k}$ to be preserved and strictly positive elsewhere.

With a given $\alpha_{k}$, one associates $P_{\alpha_{k}}(\alpha)=-\alpha_{k}+\alpha$ whose square: $P_{\alpha_{k}}^{2}(\alpha)=\alpha_{k}^{2}-2 \alpha_{k} \alpha+\alpha^{2}$ is then such that $P_{\alpha_{k}}^{2}(\alpha)>0, \forall \alpha \neq \alpha_{k}$ and $P_{\alpha_{k}}^{2}\left(\alpha_{k}\right)=0$. But $P_{\alpha_{k}}^{2}(\alpha)=$ $f_{k}^{T_{k}} v(\alpha)$ with

$$
f_{k}^{T}=\left[\begin{array}{lllllll}
\alpha_{k}^{2}-2 \alpha_{k} & 1 & 0 & 0 & \ldots & 0
\end{array}\right]
$$

and the vector $d^{T}=\left[\begin{array}{llllll}1 & 0 & 0 & 0 & \ldots & 0\end{array}\right]-f_{k}^{T}$ then satisfies conditions (5) if $\bar{A}_{o}=\left[v\left(\alpha_{k}\right)\right]$.

If there are $q$ columns in $\bar{A}_{o}$, one proceeds similarly and one builds the polynomial $P^{2}(\alpha)=\prod_{1}^{q} P_{\alpha_{k}}^{2}(\alpha)$. This polynomial has degree $2 q$, the associated vector $f$ has $2 q+1$ non-zero components and so has the sought for vector $d$. The bound (6) then follows by writing that the number of non-zero components in $d$ has to be smaller than or equal to the dimension $n$ of $d$.

Note that the bound (6) does not depend on $m$ the number of columns in $A$ and holds for both normalized or unnormalized $v(\alpha)$ columns (3) since the proof remains valid if the columns are normalized.

To fix ideas, we assumed that the $\alpha_{i}$ 's are equispaced in $[-1,1[$ but this is nowhere used in the proof and can be relaxed. On the contrary the assumption on the sign of the weights $(x \geq 0)$ is essential in our proof and seems necessary if one wants to improve the bound from (2) to (6) in the linear programming context.

\subsection{Comments}

- The necessary and sufficient bound on $\left\|x_{o}\right\|_{0}$ for $x_{o}$ to be the unique sparsest solution to $A x=A x_{o}$ (true sparsity and not just $\ell_{1}$-sparsity) is easy to obtain in this case. Since 
for (n, m)-dimensional Vandermonde matrices with distinct columns all sets of $n$ column-vectors are linearly independent, the vectors in the kernel of $A$ have at least $n+1$ nonzero components and it follows that the expected bound is $\left\|x_{o}\right\|_{0} \leq\left\lfloor\frac{n}{2}\right\rfloor$. It is identical to (6) for odd $n$ and slightly better for even $n$. This may be the price to pay (in addition to the sign constraint) to recover $x_{o}$ without an exhaustive combinatorial search.

- The bound (6) is also the one, one obtains in a different context and for a different recovery algorithm using result$\mathrm{s}$ from realization theory developed in systems theory [11]. The problem there is to recover the discrete time transfer function associated with a given impulse response. A sketch of the corresponding reasoning is as follows. A discrete time first order system with pole equal to $\alpha_{i}$ has a partial impulse response proportional to $v\left(\alpha_{i}\right)$ and the square Hankel matrix built with the $n$ samples of the response is of order $\left\lfloor\frac{n+1}{2}\right\rfloor$ and rank one. If $\left\|x_{o}\right\|_{0}=q$, then the components in $b=A x_{o}$ are seen as the first $n$ samples of the impulse response of a system built with $q$ first order systems in parallel and the rank of the order $\left\lfloor\frac{n+1}{2}\right\rfloor$ square Hankel matrix built with the $n$ samples in $b$ has rank $\min \left(q,\left\lfloor\frac{n+1}{2}\right\rfloor\right)$. If $q \leq\left\lfloor\frac{n+1}{2}\right\rfloor-1=\left\lfloor\frac{n-1}{2}\right\rfloor$ the Hankel matrix is rank deficient and one can show that the polynomial associated with any vector in its kernel has the $q$ values $\alpha_{i}$ as roots.

The algorithm to be used to recover the $\alpha_{i}$ 's, searching for eigenvectors and the roots of a polynomial, is now more complex and of a quite different nature than solving a linear program, but in this context there is no discretization involved $\left(\alpha_{i} \in \mathbb{R}\right)$ and the signs of the weights need not be constrained to be positive.

\section{THE REAL FOURIER MATRIX CASE}

\subsection{Sufficient condition for recovery}

Now the (n,m)-matrix $A$ has $m$ columns denoted $s(\omega)$ defined in (4) with $\omega$ taking $m$ distinct values for instance on a regular grid in $(0, \pi)$. The sufficient condition we will get is the same as the one given in Lemma 2 for Vandermonde matrices.

Lemma 2' Lemma 2 also holds when $A$ is an (n,m)-realFourier-matrix with $m \geq n$ distinct columns.

The proof is similar in its philosophy but different in its implementation. The polynomial $P_{\omega_{k}}(z)$ that is zero for $z=e^{ \pm i \omega_{k}}$ is $P_{\omega_{k}}(z)=\left(z-e^{i \omega_{k}}\right)\left(z-e^{-i \omega_{k}}\right)=z^{2}-$ $2 \cos \omega_{k} z+1$. And we take now the square of the modulus of this polynomial on the unit circle to guaranty that it will be zero for $z=e^{ \pm i \omega_{k}}$ and strictly positive elsewhere.

$$
\begin{aligned}
& \left|P_{\omega_{k}}(z)\right|_{z=e^{i \omega}}^{2}=P_{\omega_{k}}(z) P_{\omega_{k}}\left(z^{-1}\right) \\
& \quad=2+4 \cos ^{2} \omega_{k}-4 \cos \omega_{k}\left(z+z^{-1}\right)+\left(z^{2}+z^{-2}\right) \\
& \quad=2\left(1+2 \cos ^{2} \omega_{k}-4 \cos \omega_{k} \cos \omega+\cos 2 \omega\right)
\end{aligned}
$$

With this polynomial one associates the vector

$$
g_{k}^{T}=\left[\begin{array}{llllll}
1+2 \cos ^{2} \omega_{k} & -4 \cos \omega_{k} & 1 & 0 & 0 & \ldots .
\end{array}\right]
$$

which is such that $g_{k}^{T} s\left(\omega_{k}\right)=0$ and $g_{k}^{T} s(\omega)>0 \quad \forall \omega \neq$ $\omega_{k}$ and hence the vector $d^{T}=\left[\begin{array}{llllll}1 & 0 & 0 & 0 & \ldots & 0\end{array}\right]-g_{k}^{T}$ which satisfies (5) when there is just the column $s\left(\omega_{k}\right)$ to be isolated. If there are $q$ columns to be isolated, one proceeds as above and builds the reciprocal polynomial $\prod_{1}^{q}\left|P_{\omega_{k}}(z)\right|^{2}$ and the associated $g$ and $d$-vector will have $2 q+1$ nonzero components and the constraint $2 q+1 \leq n$ leads to the announced bound on $q=\left\|x_{o}\right\|_{0}$.

\subsection{Comments}

- The comments are similar to those made for Vandermonde matrices. The fact that any set of $n$ distinct columns of the form (4) are independent is less well known than in the Vandermonde case but can be proven by noting that $s(\omega)=$ $T v(\cos (\omega))$ with $T$ a non-singular lower-triangular matrix independent of $\omega$ whose lines contain the coefficients of the Tschebyscheff polynomials and the result then follows from the Vandermonde case. Hence the bound $\left\|x_{o}\right\|_{0} \leq\left\lfloor\frac{n}{2}\right\rfloor$ that is necessary and sufficient for the $\ell_{o}$-sparsity. One can note that this same transformation $T$ holds between the vectors $f_{k}$ and $g_{k}$ introduced above.

- The bound (6) is the same one obtains using a powerful theorem by Caratheodory [12] which says that given $n-1$ complex numbers $\left\{b_{2}, b_{3}, \ldots, b_{n}\right\}$, not all zero, there exists an unique sparsest decomposition satisfying :

$$
b_{j+1}=\sum_{1}^{q} x_{p} e^{i j \omega_{p}}, \quad j=1, \ldots, n-1
$$

with $\left.\left.x_{p}>0, \omega_{p} \neq \omega_{k} \in\right]-\pi, \pi\right]$ and $q \leq n-1$.

If one specializes this theorem to real numbers $b_{j}$, one observes that which each $\omega_{p} \neq 0$ or $\pi$ in the decomposition one can then associate an $\omega_{p^{\prime}}=-\omega_{p}$ with $x_{p^{\prime}}=x_{p}$. In our context, this implies that if $b=\sum_{1}^{q} x_{\ell} s\left(\omega_{\ell}\right)$ with arbitrary $\left.\omega_{\ell} \in\right] 0, \pi\left[, x_{\ell}>0\right.$ and $q \leq\left\lfloor\frac{n-1}{2}\right\rfloor$ then this decomposition is unique. One gets the same bound (6) with signconstrained weights but for arbitrary values of $\omega$ not necessarily belonging to a grid. The theorem by Caratheodory however does not tell us how to recover the decomposition. The corresponding recovery part has been proposed by Pisarenko [13] and, as for the method based on realization theory sketched in section 3.2, requires to compute the roots of a polynomial.

- The method based on realization theory proposed in section 3.2 also applies to the present real Fourrier case, but since each oscillator (sinusoid) induces a rank two in the Hankel matrix the bound on $\left\|x_{o}\right\|_{0}$ one gets is twice lower than (6). This bound can in fact further be improved in the present context and in anycase this approach imposes no sign-constraint on the weights. 


\section{REMARKS ON THE UNIQUENESS OF THE SOLUTION}

The results we established (Lemma 2 and 2') are valid for both normalized or un-normalized columns in $A$. In the last case, see (3),(4), the first row of $A$ is filled with ones and the first equation in $A x=b(\mathrm{LP}+)$ is $\mathbf{1}^{T} x=b(1)$ where $b(1)$ denotes the first component of $b$. Since the criterion in (LP+) is precisely $\min \mathbf{1}^{T} x$, this means that all admissible points have the same cost $b(1)$. Since we proved uniqueness of the optimal solution, we actually established uniqueness of the admissible points.

Lemma 3: For $A$ an $(\mathrm{n}, \mathrm{m})$-Vandermonde or real-Fourier matrix with distinct columns, the solution to $\{A x=b, x \geq$ $0\}$ is unique if $b=A x_{o}$ with $x_{o} \geq 0$ and

$$
\left\|x_{o}\right\|_{0} \leq\left\lfloor\frac{n-1}{2}\right\rfloor
$$

where $\lfloor z\rfloor$ denotes the nearest integer that is $\leq z$.

And indeed one can reach the same conclusion by observing that the vector $f$ (and $g$ ) we introduced in the proofs of Lemma 2 and 2' verifies the following proposition:

Proposition: The solution $x_{o}$ of $\{A x=b, x \geq 0\}$ with $b=A x_{o}=\bar{A}_{o} \bar{x}_{o}$ is unique if

$$
\exists f \ni \quad \bar{A}_{o}^{T} f=0 \text { and } a_{j}^{T} f>0 \forall a_{j} \notin \bar{A}_{o}
$$

with $\bar{A}_{o}$ is a full column rank matrix.

This result applies only for $\left\|x_{o}\right\|_{0}<n$, since otherwise $f=0$. It is a consequence of the following lemma we will prove.

Lemma 4: The j-th component of all points in the set, assumed to be non-empty, $\{A x=b, x \geq 0\}$ is zero if and only if $\exists f \quad \exists \quad b^{T} f=0, A^{T} f \geq 0, a_{j}^{T} f>0$.

Proof: To establish the necessity of the condition, one introduces the linear program

$$
\min -e_{j}^{T} x \text { subject to } A x=b, x \geq 0
$$

with $e_{j}$ is the $\mathrm{j}$-th column of the identity matrix. Its dual is $\max b^{T} d$ subject to $A^{T} d \leq-e_{j}$.

With $x_{o}$ the optimum of the primal, that exists by assumption and has cost zero, one can then associate $d_{o}$ an optimum of the dual which satisfies $b^{T} d_{0}=-e_{j}^{T} x_{o}=0$, $A^{T} d_{0} \leq 0$ and $a_{j}^{T} d_{0} \leq-1$. One then takes $f=-d_{o}$ to establish the result. The condition is sufficient since it implies $f^{T} A x=f^{T} b=0$. But $f^{T} A x=0$ with $f^{T} A \geq 0$ and $x \geq 0$ in turn implies that $e_{j}^{T} x=0$ whenever $f^{T} a_{j}>0$. $\square$

The proposition then follows from Lemma 4 and the assumption that $\bar{A}_{o}$ is a full column rank matrix.

\section{CONCLUSIONS}

We have considerably improved the bound (2) known for arbitrary matrices by exploiting the specific structure of the Vandermonde and real Fourier matrix. The new bound (6) is independent of the number $m$ of components in the dictionary but holds only under the assumption that the weights are sign-constrained. It is our impression that this restriction cannot be removed in case the recovery has to be achieved by solving a linear program.

This analysis is also of interest because it allows to assess the resolution or separation properties of estimation methods based on this kind of approaches. The fact that the bound (6) is independent of $m$ indicates that it is possible to develop high-resolution methods. This possibility has already been exploited for instance in [14] where the linear program is replaced by a quadratic program.

We have also mentioned for the same two specific situations the existence of other recovery algorithms and in each case indicated the corresponding bounds and potential sign-restrictions.

\section{REFERENCES}

[1] D.L. Donoho and X. Huo. Uncertainty principles and ideal atomic decomposition. IEEE Trans. on I.T., 47, 11, 2845-2862, Nov. 2001

[2] M. Elad an A.M. Bruckstein. A generalized uncertainty principle and sparse representation in pairs of bases. IEEE Trans. on I.T., 48, 9, 2558-256, Sept. 2002.

[3] R. Gribonval and M. Nielsen. Sparse representations in unions of bases. IEEE Trans. on I.T. 49, 12, 3320-3325, Dec. 2003.

[4] J.J. Fuchs. More on sparse representations in arbitrary bases. IEEE Trans. on I.T. 50, 6, 1341-1344, June 2004 see also 13th Ifac-Ifors symposium on Identification and system Parameter Estimation, pp. 1357-1362, Rotterdam,

[5] J.A. Tropp, "Greed is good: Algorithmic Results for Sparse Approximations," ICES Report 0304, UT-Austin, Feb. 2003, to appear in IEEE Trans. on I.T.

[6] D. Malioutov, M. Cetin and A.S. Willsky "Optimal sparse representations in general overcomplete bases," IEEE ICASSP, vol II, 793-796, Montreal, May 2004.

[7] B. K. Natarajan. Sparse approximate solutions to linear systems. SIAM J. Comput., 24, 21, 227-234, April 1995.

[8] T. Strohmer and R. Heath, "Grassmanian frames with applications to coding and communications," to appear in Appl. Comp. Harm. Analysis.

[9] A. Gilbert, S. Muthukrishnan and M.J. Strauss, "Approximation of functions over redundant dictionaries using coherence," 14th ACM-SIAM Symposium on Discrete Algorithms, (SODA'03), Jan. 2003.

[10] D. G. Luenberger. Introduction to linear and nonlinear programming. Addison Wesley, 1973.

[11] T. Kailath. Linear systems. Prentice Hall, 1980.

[12] U. Grenander and G. Szego. Toeplitz forms and their applications. Berkeley, Univ. Calif. Press, 1958.

[13] V.F. Pisarenko. The retrieval of harmonics from a covariance function. Geophys. J. Roy. Astron. Soc., 347-366, 1973.

[14] J.J. Fuchs, "On the application of the global matched filter to DOA estimation with uniform circular arrays," IEEE-T-SP, vol. 49, p. 702-709, avr. 2001. IEEE Signal Processing Society 2003 Best Paper Award. 